\title{
The characterization of blood flow changes in mouse tumor during Photofrin-based photodynamic therapy by using the color Doppler ultrasonography
}

\author{
KARMEN DUBRETA $^{1}$, SINISA IVANKOVIC ${ }^{2}$, ARIJANA LOVRENCIC-HUZJAN ${ }^{3}$, \\ MARIJANA BOSNAR-PURETIC ${ }^{3}$, RANKO STOJKOVIC ${ }^{2}$ and MISLAV JURIN ${ }^{2}$ \\ ${ }^{1}$ Department of Obstetrics and Gynecology, University Clinic for Gynecology and Obstetrics, Petrova 13, \\ HR-10000 Zagreb; ${ }^{2}$ Department for Molecular Medicine, Rudjer Boskovic Institute, P.O. Box 180, HR-10002 Zagreb; \\ ${ }^{3}$ University Department of Neurology, Sestre Milosrdnice University Hospital, Vinogradska c. 29, HR-10000 Zagreb, Croatia
}

Received April 1, 2009; Accepted July 7, 2009

DOI: $10.3892 /$ or_00000562

\begin{abstract}
Changes in blood flow velocity through the tumors can induce damage of tumor microcirculation and thus may contribute to the final destruction of tumor masses after photodynamic therapy (PDT). The aim of this study was to evaluate the blood flow changes in a SCCVII mouse carcinoma during Photofrin-based photodynamic therapy by analyzing several quantitative spectral Doppler parameters [maximum systolic flow velocity (Vmax), end diastolic velocity (Vmin), resistance index (RI) and pulsatile index (PI)] by using the color Doppler ultrasonography. Blood flow velocities were recorded immediately prior to tumor illumination $(0 \mathrm{~h})$ and then 2 and $24 \mathrm{~h}$ after the illumination. Statistically significant increase in diastolic blood velocity (Vmin) with a corresponding decline in RI and PI was recorded in tumors of the Photofrin-injected mice prior to tumor illumination. However, $2 \mathrm{~h}$ after the illumination a pronounced decrease in both Vmin and Vmax was obtained. There were no changes of these parameters in controls at different times during determination. The observed changes of spectral Doppler parameters in tumors from the PDT group point to the transition of tumor blood vessels from the relaxation state recorded before tumor illumination into a state of increased contraction after the activation of Photofrin by light. Pronounced changes in tumor blood vessel tone might be an additional stress for such vessels leading to their ultimate destruction.
\end{abstract}

Correspondence to: Dr Sinisa Ivankovic, Department for Molecular Medicine, Rudjer Boskovic Institute, P.O. Box 180, HR10002 Zagreb, Croatia

E-mail: sivankov@irb.hr

Key words: photodynamic therapy, photofrin, tumor, blood flow velocity, vascular resistance, color Doppler ultrasound

\section{Introduction}

Photodynamic therapy (PDT) is one of the approaches used in controlling tumor growth. Photoactive components in combinations with the light of adequate wavelength are responsible for tumor destruction through the action of highly reactive oxygen intermediates and host immune system activation, or by damaging tumor vascular structures (1). Further, it should be kept in mind that vessel structures are more vulnerable in tumor than in normal tissue $(2,3)$. It has been shown that PDT induces damage of tumor microcirculation acting either directly on the structure of blood vessels, or by changing blood flow rate (4-6). Both could be responsible for additional tumor destruction in PDT. In fact, while it seems pretty clear that the PDT eventually results in excluding the tumor vasculature due to reducing the blood flow through the tumor, platelet aggregation and the constriction of tumor vessels $(4,5,7,8)$ there is still a lack of information on the changes in the blood flow through tumors immediately before or shortly following tumor exposure to the light during a photodynamic treatment.

Various imaging techniques have been used to assess tumor blood flow during PDT. In contrast to invasive methods such as intratumoral laser Doppler velocimeter (9) and intravital fluorescence microscopy (10), color Doppler ultrasonography (CDU) represents a non-invasive method to estimate in real-time the blood flow velocity in arterioles (11). The CDU as a readily accessible, easy to use, quick and safe method have got a significant role in diagnosis and treatment assessments. In addition, the method allows us to quantify the tumor microcirculation several times in the same patient. Numerous clinical studies have shown the usefulness of CDU in the monitoring of the effects of chemotherapy (12) and radiotherapy (13), but only one study, which surveyed the effects of photodynamic therapy in patient with age-related macular degeneration, has been recently published (14). However, there are no data available about the use of CDU either in the monitoring of efficiency of PDT in the cancer treatment or in the characterisation of vascular changes in tumors during PDT. 
In this study, by using the CDU, we analysed several quantitative spectral Doppler parameters (maximal and minimal velocities of blood flow and resistance and pulsatile indices) in order to characterize the vascular response in tumors of the Photofrin-injected tumor-bearing mice at particular times prior to and after the exposure to light. This is the first study on such an investigation during a photodynamic treatment. Given the complexity of vascular responses in tumors during $\mathrm{PDT}$ as well as taking into account that the therapeutic success of PDT is dependent on the degree of damage to tumor blood vessels, better knowledge of the sequence of events that preceded the destruction of tumor vasculature can contribute to establishing a more efficient PDT protocol for cancer treatment.

\section{Materials and methods}

Animals and tumor model. $\mathrm{C} 3 \mathrm{Hf} / \mathrm{Bu}$ male mice obtained from Rudjer Boskovic Institute's breeding colony were used in the study. The animals were 3 months old at the beginning of the experiments and had access to food (4RF 21 GLP Mucedola srl, Italy) and water ad libitum. The experiments were performed according to the ILAR Guide for the Care and Use of Laboratory Animals, Council Directive (86/609/EEC) and Croatian animal protection law (NN 135/2006). Tumor model of SCCVII mouse carcinoma was used. The mice were subcutaneously injected into the shaved right thigh with $5 \times 10^{5}$ SCCVII mouse carcinoma cells in $100 \mu 1$ of RPMI using a tuberculin syringe and a 25 -gauge needle. Tumors were measured by callipers (Lange Skin fold Calliper, Cambridge Scientific Industry, USA) and tumor volumes were calculated by the formula: length $\mathrm{x}$ width $\mathrm{x}$ depth $\mathrm{x} \pi / 6$.

Photosensitizer and source of light. The photosensitizer, Photofrin II (Quadra Logic Technologies, Vancouver, Canada), was dissolved in $5 \%$ dextrose at a concentration of $1 \mathrm{mg} / \mathrm{ml}$. It was administered intraperitoneally to the animals at the dose of $20 \mathrm{mg} / \mathrm{kg}$ at $48 \mathrm{~h}$ before tumor illumination. As the source of light, we used a $250 \mathrm{~W}$ halogen lamp equipped with the focusing system and a set of optical cut-on and cut-off filters which eliminated all light wavelengths outside the $600-700 \mathrm{~nm}$ interval.

Tumor treatment. Eight days after tumor cell inoculation, when the tumors reached 7-8 $\mathrm{mm}$ in diameter, animals were divided in two groups, the control and photodynamic therapy (PDT) group. Seven mice were used in each group. Mice in the PDT group were intraperitoneally injected with Photofrin in a dose of $20 \mathrm{mg} / \mathrm{kg}$ and two days after the Photofrin injection, the legs with tumors received $630 \mathrm{~nm}$-wavelength light treatment over a period of $20 \mathrm{~min}$. The control mice received $5 \%$ dextrose (no Photofrin) and two days later, the same light treatment as the mice from the PDT group. In the period after the Photofrin administration mice from both experimental and control groups were kept in a dark chamber.

Determination of spectral Doppler blood flow parameters in tumors. The determination of spectral Doppler blood flow parameters in tumors was performed two days after the Photofrin injection i.e. 10 days after the tumor cell inoculation.
At that point, the tumors were $\sim 10 \mathrm{~mm}$ in the longest diameter and blood vessels were detectable on color Doppler ultrasound. Tumors were imaged with an Acuson 128 XP machine (Mountain View, CA, USA) using a $7 \mathrm{MHz}$ linear array probe directly before the light treatment and then 2 and $24 \mathrm{~h}$ after the tumor illumination. The total examination time per animal was $\sim 10 \mathrm{~min}$. Specially designed holders were used for immobilizing the mice during PDT illumination without anaesthesia. The ultrasound probe was covered with copious amounts of gel so as to not apply significant external pressure on the tumor, which might alter blood flow. When tumor vessels were detected by color Doppler, flow velocity waveforms were recorded in the pulsed Doppler mode by placing the sample volume over the colored vessels. Maximum systolic flow velocity (Vmax) and end diastolic velocity (Vmin) were recorded in the tumor vessels and the resistance index (RI) and pulsatile index (PI) were computed using the velocity data. The resistance index was computed according to the Pourcelot's formula: $\mathrm{RI}=(\mathrm{V} \max -\mathrm{Vmin}) / \mathrm{Vmax}$, while the pulsatile or impedance index was calculated using the formula PI $=($ Vmax $-V m i n) / V m e a n$, where Vmean designates the mean blood flow velocity.

Statistical analysis. Data are expressed as mean \pm standard deviations. Intergroup comparison between PDT and controls in the respective measuring point was performed by the t-test for independent samples. To analyze the differences between measuring points in the same group, ANOVA for repeated measures was used. If ANOVA showed significant differences, a paired t-test with Bonferroni correction for multiple comparisons was used to locate the point of significance (15). All statistical analyses were performed using the SigmaStat statistical software. P-values $<0.05$ were considered as statistically significant.

\section{Results}

The mice bearing SCCVII carcinoma transplanted in the right flank $(\mathrm{n}=7)$ were injected i.p. with $20 \mathrm{mg} / \mathrm{kg}$ of Photofrin when the tumors were $\sim 8-9 \mathrm{~mm}$ in diameter. Forty-eight hours later the tumors of the mice from control (no Photofrin injected) and PDT groups were illuminated with a $630 \mathrm{~nm}$-wavelength for $20 \mathrm{~min}$. The measurements of blood flow parameters were performed immediately prior to $(0 \mathrm{~h})$ and then 2 and $24 \mathrm{~h}$ after tumor illumination.

Changes in the end diastolic velocity (Vmin) and the maximum systolic velocity (Vmax) are presented in Figs. 1 and 2, respectively. At the time 0 i.e. just prior to light exposure, the values of Vmin in the PDT group were significantly higher $(\sim 37 \%)$ than in the control group. In contrast to Vmin, there were no differences in the maximum systolic velocity (Vmax) between these two groups (Fig. 2) at the time 0 . Two hours later a significant decrease of both Vmin $(45 \%)$ and $V \max (\sim 35 \%)$ was observed in tumors of PDT-treated mice. At $24 \mathrm{~h}$, there were no differences in Vmin and Vmax between the PDT-treated mice and their controls. There were no significant changes of these parameters in controls at different times during determination.

Prior tumor exposure to light (the time 0), as a result of the pronounced increase of the diastolic blood flow velocity 


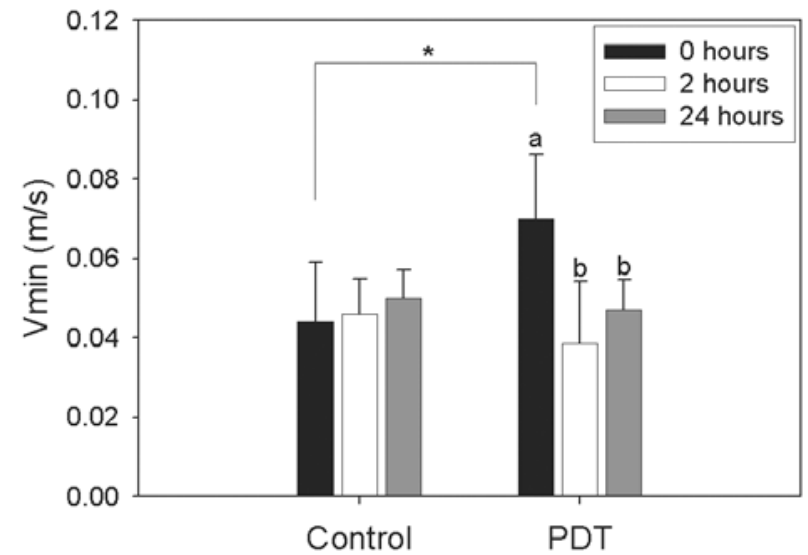

Figure 1. End diastolic velocity (Vmin) in the control and PDT-treated groups prior to $(0 \mathrm{~h})$ and 2 and $24 \mathrm{~h}$ after local tumor illumination. The $(*)$ indicates significant difference between control and PDT group for particular time-point. The letters $\mathrm{a}$ and $\mathrm{b}$ above bars indicate significant differences between measuring points in the same group; the bars that do not have common letters are significantly different.

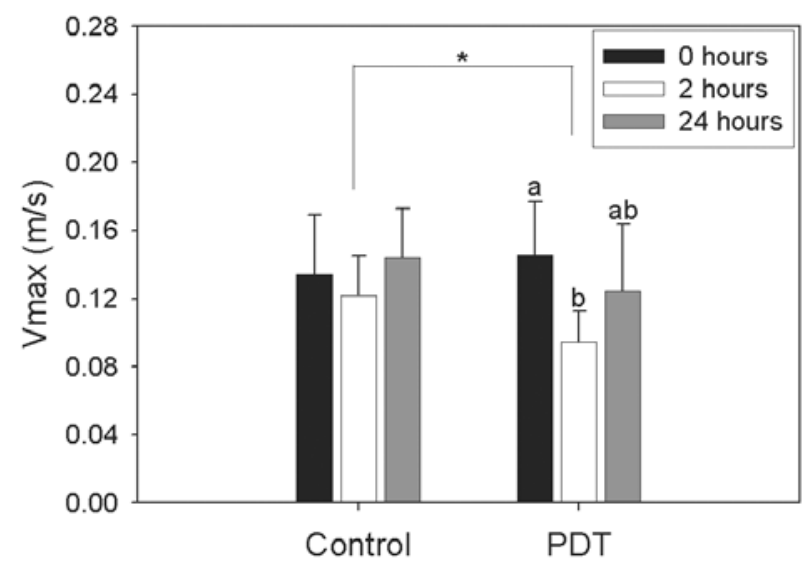

Figure 2. Maximum systolic flow velocity (Vmax) in the control and PDTtreated groups prior to $(0 \mathrm{~h})$ and 2 and $24 \mathrm{~h}$ after local tumor illumination. The $(*)$ and the letters a and b (see explanation given in Fig. 1).

(Vmin), the resulting pulsatile index (PI, Fig. 3) was $\sim 40 \%$ lower $(\mathrm{P}<0.05)$ in the Photofrin-injected mice. However, $2 \mathrm{~h}$ after the baseline measuring, an increase of PI was observed, but the difference obtained was not at the statistically significant level. At $24 \mathrm{~h}$, the values of PI increased and were similar to its values in the control animals. Further, as presented in Fig. 4, the resistance index (RI) also significantly decreased at the time 0 , but that reduction was somewhat lower than it was for the PI (22 vs. $40 \%$ ).

\section{Discussion}

Presented results pointed to pronounced changes in blood flow parameters in the tumor of Photofrin-treated mice prior to and after local light application. By using color Doppler ultrasonography the blood flow velocity was determined in tumor blood vessels. A significant increase of blood flow, especially the diastolic blood flow velocity, was recorded at $48 \mathrm{~h}$ following i.p. Photofrin injection, but $2 \mathrm{~h}$ after tumor

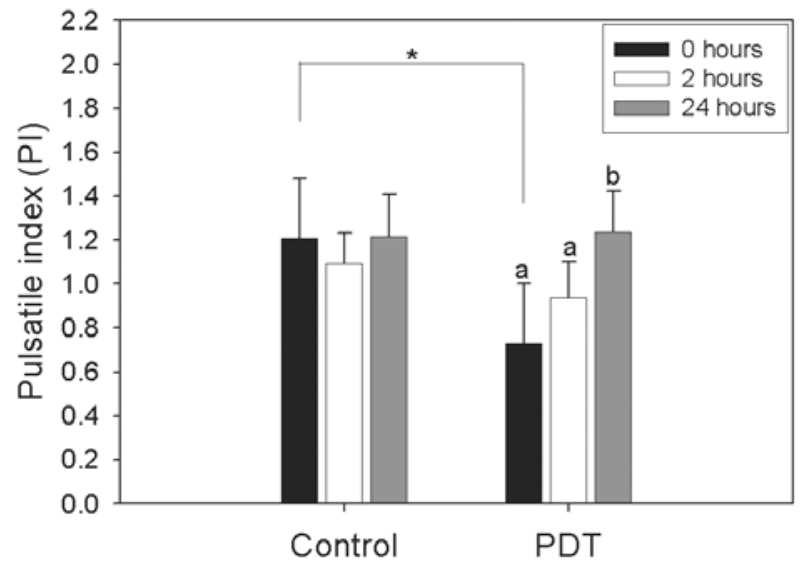

Figure 3. Pulsatile index (PI) in the control and PDT-treated groups prior to $(0 \mathrm{~h})$ and 2 and $24 \mathrm{~h}$ after local tumor illumination. The $(*)$ and the letters ( $\mathrm{a}$ and $\mathrm{b}$ (see explanation given in Fig. 1).

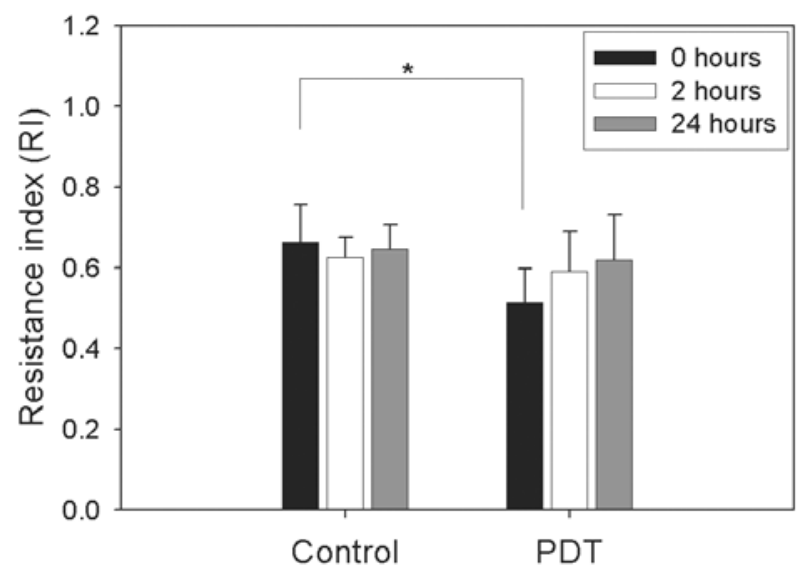

Figure 4. The resistance index (RI) in the control and PDT-treated groups prior to $(0 \mathrm{~h})$ and 2 and $24 \mathrm{~h}$ after tumor illumination. The $(*)$ indicates significant difference between control and PDT group for particular time-point.

illumination a pronounced decrease in blood flow velocity was obtained.

The observation that the application of Photofrin resulted in a significant increase of blood flow in tumors $48 \mathrm{~h}$ following injection deserves special attention. There are at least two possible explanations for the recorded increase in blood flow under the influence of non-activated Photofrin: i) vasodilatation of the functionally active mature tumor blood vessels and ii) opening of the previously closed arteriovenous shunts and/or blood vessels.

Taking into account that the observed increase of diastolic blood flow velocity was accompanied with a significant decrease of pulsatile and resistance indices, in addition to the fact that these indices are good indicators of changes in vascular resistance $(16,17)$, it can be assumed that the nonactivated Photofrin, by causing vasodilatation, reduces the vascular resistance and thus increases the blood flow through the tumor. With respect to the specifics of tumor microcirculation, at this point, a question araises about the ability of tumor blood vessels to respond by changing their muscle tone on the stimulation with vasomodulatory substances. 
Indeed, newly formed tumor microvessels lack smooth muscle layers and are therefore unable to provide autoregulation. However, pre-existing normal vessels encased by tumor have smooth muscle layers and therefore can functionally respond to different vasoactive stimuli. Furthermore, several studies have provided indirect evidence that at least a proportion of the tumor vasculature can respond to vasomodulatory drugs and thereby increase oxygenation and drug delivery to tumors $(18,19)$. Thus, the increase in blood flow velocity recorded in this study can be also, at least partly, attributed to the vasodilatation of such pre-existing mature tumor blood vessels.

Taking into account the fact that only $20-80 \%$ of tumor vessels are actually perfused (20) as well as that increase of blood flow accompanied by a high diastolic flow as observed in our study, is a characteristic Doppler finding for the arteriovenous shunts (21), we can also assume opening of the previously closed arteriovenous shunts and/or blood vessels. Due to the above-mentioned changes in blood flow velocity/ vascular resistance, more and more blood enters into the tumor areas that were hypoxic or ischemic. As a consequence of such an extensive tumor circulation, the numerous tumor areas become not only better oxygenated, but also overfilled with the photosensitizer (22). Increased tumor oxygenation could have a benefit not only for the PDT itself $(23,24)$, but also for other modalities of antitumor therapy whose therapy outcome depends on intra-tumor oxygen concentration as it is the case, for example, in radiotherapy (25). Therefore, the obtained results suggest the possible use of Photofrin as a vasoactive substance for increasing tumor perfusion and oxygenation in antitumor therapies that use the so-called 'pro-vascular' approach as the first component in antitumor treatment (26).

Furthermore, the reduction of blood flow velocity along with the increase of RI and PI, which was observed after the activation of Photofrin by light, points to the increase in vascular resistance in tumors from the PDT group. This decrease in blood flow velocity could be, at least partly, explained by a vasoconstriction of 'functionally' active blood vessels inside the tumors (26), although other physiological responses initiated after endothelial cell damage, such as platelet aggregation, the release of vasoactive molecule as a consequence of acting either the photoactive substance itself or some of the components produced after its photoactivation, leukocyte adhesion and increase in vascular permeability $(4,5,7,8)$, must also be taken into account. In this context as well as in line with the fact that after the activation of Photofrin by the light an intensive production of oxygen radicals was observed (1), it can be assumed that these reactive oxygen species represent a switch causing the transition of tumor blood vessels from the relaxation state recorded before tumor illumination into a state of increased contraction after the illumination. Similarly to our results, Yu et al have detected a decrease in relative blood flow in fibrosarcoma murine tumors at 3 and $6.5 \mathrm{~h}$ after PDT (8), while Wieman et al found markedly reduction of tumor blood flow in rat chondrosarcomas even at $5 \mathrm{~min}$ after the tumor illumination (9). Engbrecht et al have also demonstrated a significant reduction in blood flow in a human sarcoma xenograft model at $6 \mathrm{~h}$ after the Photofrin-mediated PDT (4).
Since the tumor blood vessels are fragile and therefore much more vulnerable than normal blood vessels $(2,3)$, pronounced changes in vascular dynamic as it was showed in this study, could represent an additional mechanical stress for such weak vascular structures and can lead to their ultimate destruction. In addition to the vascular changes, a direct destruction of tumor cells by apoptosis $(4,27)$, or the influence of activated host immune system (28), could be also expressed after proper light application to the tumor.

In conclusion, the results obtained have confirmed that the technique of color Doppler ultrasonography can be successfully applied for the characterization of vascular responses during PDT. Pronounced blood flow velocity/vascular resistance changes in tumor vessels of Photofrin-treated mice were observed both prior to as well as after local light application. Considering that vessel structures are more vulnerable in tumors than in normal tissues, frequent and pronounced changes in vascular tone of the tumor blood vessels may be important for their ultimate destruction and therefore may contribute to the final destruction of a tumor mass after PDT treatment. Moreover, the pronounced vasodilatatory effect of non-activated Photofrin obtained in this study suggests the possible use of this photoactive substance as a 'pro-vascular' agent for increasing the blood flow through the tumor in the combined antitumor therapeutic modalities.

\section{Acknowledgements}

The authors gratefully acknowledge the Ministry of Science, Education and Sport, Republic of Croatia, for the financial support for the Projects 098-0982464-2390.

\section{References}

1. Dougherty TJ, Gomer CJ, Henderson BW, Jori G, Kessel D, Korbelik M, Moan J and Peng Q: Photodynamic therapy. J Natl Cancer Inst 90: 889-905, 1998.

2. Holash J, Wiegand SJ and Yancopoulos GD: New model of tumor angiogenesis: dynamic balance between vessel regression and growth mediated by angiopoetins and VEGF. Oncogene 18: 5356-5362, 1999 .

3. McDonald DM and Foss AJE: Endothelial cells of tumor vessels: abnormal but not absent. Cancer Metastasis Rev 19: 109-120, 2000.

4. Engbrecht BW, Menon C, Kachur AV, Hahn SM and Fraker DL: Photofrin-mediated photodynamic therapy induces vascular occlusion and apoptosis in a human sarcoma xenograft model. Cancer Res 59: 4334-4342, 1999.

5. Fingar VH, Kik PK, Haydon PS, Cerrito PB, Tseng M, Abang E and Wieman TJ: Analysis of acute vascular damage after photodynamic therapy using benzoporphyrin derivative (BPD). Br J Cancer 79: 1702-1708, 1999.

6. Krammer B: Vascular effects of photodynamic therapy. Anticancer Res 21: 4271-4277, 2001.

7. Garbo GM, Vicente MG, Fingar V and Kessel D: Effects of ursodeoxycholic acid on photodynamic therapy in a murine tumor model. Photochem Photobiol 78: 407-410, 2003.

8. Yu G, Durduran T, Zhou C, Wang HW, Putt ME, Saunders HM, Sehgal CM, Glatstein E, Yodh AG and Busch TM: Noninvasive monitoring of murine tumor blood flow during and after photodynamic therapy provides early assessment of therapeutic efficacy. Clin Cancer Res 11: 3543-3552, 2005.

9. Wieman TJ, Mang TS, Fingar VH, Hill TG, Reed MW, Corey TS, Nguyen VQ and Render ER: Effect of photodynamic therapy on blood flow in normal and tumor vessels. Surgery 104: 512-517, 1988.

10. He C, Agharkar P and Chen B: Intravital microscopic analysis of vascular perfusion and macromolecule extravasation after photodynamic vascular targeting therapy. Pharm Res 25: 1873-1880, 2008. 
11. Perini R, Choe R, Yodh AG, Sehgal C, Divgi CR and Rosen MA: Non-invasive assessment of tumor neovasculature: techniques and clinical applications. Cancer Metastasis Rev 27: 615-630, 2008.

12. Bramer JA, Gubler FM, Maas M, Bras H, de Kraker J, van der Eijken JW and Schaap GR: Colour Doppler ultrasound predicts chemotherapy response, but not survival in paediatric osteosarcoma. Pediatr Radiol 34: 614-619, 2004.

13. Kerimoglu U, Akata D, Hazirolan T, Ergen FB, Köse F, Ozyar E, Atahan LI and Akhan O: Evaluation of radiotherapy response of cervical carcinoma with gray scale and color Doppler ultrasonography: resistive index correlation with magnetic resonance findings. Diagn Interv Radiol 12: 155-160, 2006.

14. Turkcuoglu P, Deniz N, Koc M, Kurt J and Celiker U: The effect of photodynamic therapy on retrobulbar blood flow parameters. Clin Exp Ophthalmol 36: 39-42, 2008.

15. Sokal RR and Rohlf FJ: Biometry. W.H. Freeman and Co., New York, 1995.

16. Bude RO and Rubin JM: Relationship between the resistive index and vascular compliance and resistance. Radiology 211: 411-417, 1999

17. Yuan A, Yang PC, Lee L, Wu HD, Kuo SH, Luh KT, Chen WJ and Lin FY: Reactive pulmonary artery vasoconstriction in pulmonary consolidation evaluated by color Doppler ultrasonography. Ultrasound Med Biol 26: 49-56, 2000.

18. Song CW: Modification in blood flow. In: Blood Perfusion and Microenvironment of Human Tumors. Molls M and Vaupel P (eds). Springer-Verlag, pp193-207, 1998.

19. Gallez B, Jordan BF, Baudelet C and Misson PD: Pharmacological modifications of the partial pressure of oxygen in murine tumors: evaluation using in vivo EPR oximetry. Magn Reson Med 42: 627-630, 1999 .
20. Delorme S and Knopp MV: Non-invasive vascular imaging: assessing tumour vascularity. Eur Radiol 8: 517-527, 1998.

21. Grenier N, Basseau F, Rey MC and LaGoarde-Segot L: Interpretation of Doppler signals. Eur Radiol 11: 1295-1307, 2001.

22. Korbelik M and Krosl G: Photofrin accumulation in malignant and host cell populations of various tumours. Br J Cancer 73: 506-513, 1996.

23. Fuchs $J$ and Thiele $J$ : The role of oxygen in cutaneous photodynamic therapy. Free Radic Biol Med 24: 835-847, 1998.

24. Huang Z, Chen Q, Shakil A, Chen H, Beckers J, Shapiro H and Hetzel FW: Hyperoxygenation enhances the tumor cell killing of photofrin-mediated photodynamic therapy. Photochem Photobiol 78: 496-502, 2003.

25. Siemann DW, Warrington KH and Horsman MR: Targeting tumor blood vessels: an adjuvant strategy for radiation therapy. Radiother Oncol 57: 5-12, 2000.

26. Feron O: Targeting the tumor vascular compartment to improve conventional cancer therapy. Trends Pharmacol Sci 25: 536-542, 2004.

27. Oleinick NL, Morris RL and Belichenko I: The role of apoptosis in response to photodynamic therapy: what, where, why, and how. Photochem Photobiol Sci 1: 1-21, 2002.

28. van Duijnhoven FH, Aalbers RI, Rovers JP, Terpstra OT and Kuppen PJ: The immunological consequences of photodynamic treatment of cancer, a literature review. Immunobiology 207: 105-113, 2003. 